\title{
The Development and Concurrent Validity of a Multi- sensor-based Frailty Toolkit for In-home Frailty Assessment
}

Chao Bian ( $\nabla$ chao.bian@mail.utoronto.ca )

University of Toronto

Bing Ye

Toronto Rehabilitation Institute

Alex Mihailidis

University of Toronto

\section{Research Article}

Keywords: Frailty, Assessment, Sensors, Measurement, Validation, Agreement

Posted Date: November 19th, 2021

DOI: https://doi.org/10.21203/rs.3.rs-1082854/v1

License: (c) (i) This work is licensed under a Creative Commons Attribution 4.0 International License.

Read Full License 


\section{Abstract}

\section{Background}

Early identification of frailty is crucial to prevent or reverse the syndrome but faces challenges due to frailty's insidious onset. Monitoring behaviroal changes in real life may offer opportunities for early identifying frailty. Home-based technologies for assessing frailty have shown potentials. However, they are still in early stage of development with many types of technologies and behavioural and physical signs associated with frailty underexamined. This study presented a sensor-based system that used heterogeneous sensors and cloud technologies to monitor behavioural and physical signs of frailty from home settings. We aimed to validate the concurrent validity of the sensor system.

Methods

The sensor system consisted of motion, door, distance and mat sensors, a smart speaker, and a smart weight scale. The selection of these sensors was based on behavioural and physical signs found significantly associated with frailty. These signs included room-level physical activity, life space, sedentary behaviour, stair climbing, self-report exhaustion, and weight. Older adults' voices were included in the system design. The sensor system prototype was tested in a simulated home lab environment with nine young, healthy participants. Cohen's kappa and Bland-Altman Plot statistical methods evaluated the agreements between the sensor and ground truth measurements.

Results

Excellent concurrent validity was achieved for the smart speaker ( $100 \%$ agreement), the motion sensor (a kappa value of $\mathrm{k}=0.938)$, the door sensor ( $87.5 \%$ agreement, $100 \%$ if excluding outliers), the mat sensor (mean difference of $-0.286,95 \%$ limits of agreement) and the distance sensor (mean difference of 0.526 , $95 \%$ limits of agreement). The smart weight scale and traditional weight scale had a significant difference in weight measurements. However, bivariate correlation showed a strong, positive correlation between the two measurements $(r=0.942, n=24, p<0.001)$.

Conclusions

Overall, FT is reliable for monitoring physical and behavioural signs of frailty in home settings. The ambient sensors and the smart speaker in FT showed excellent concurrent validity, while the smart weight scale needs further validation. This work laid the ground for the next step of the research to test the toolkit with frail and non-frail older adults to validate the system's clinical effectiveness.

\section{Introduction}

Background 
According to World Population Prospects: the 2019 Revision, by 2050, one in six people in the world will be over age 65 (16\%), up from one in 11 in 2019 (9\%) [1]. Older adults are at a high risk of disability, longterm hospitalization, unfavourable discharge, and death after injury, but age itself is a poor indicator of risk because of the heterogeneity of older adults [2-4]. The concept of "frailty" identifies older adults with low physiological reserves and vulnerability to illness and high risk of disability, institutionalization, and death $[5,6]$. People in a frailty state are vulnerable to stressful situations and gradually decline towards dependence if the frailty state is not detected early enough. As a consequence, frail people are unable to live at home independently without supervision. This increase of dependency on the aging population will have a significant economic impact due to the healthcare cost for the frail population [7]. For instance, the incremental one-year health care costs associated with frailty went from $\$ 10,845$ to $\$ 12,360$ in Canada [8]. In Germany, progression from non-frailty to frailty caused an increase in total health care costs [9]. The costs increased by approximately 54 and $101 \%$ if 3 and 4 or 5 frailty symptoms were present.

Despite the adverse outcomes, frailty can be prevented or remedied [10]. Studies have shown that frailty is a dynamic process characterized by frequent transitions between frailty states over time $[5,6]$. Fried et al. defined three frailty states based on the number of phenotypic criteria, such as slowness, weakness, or shrinking that a person meets [6]. A person is frail if meeting three to five phenotypic criteria, pre-frail if meeting one or two criteria, and non-frail if none of the phenotypic criteria are present. The dynamic transitions between frailty states mean that pre-frail people can transition to non-frail with intervention. The likelihood of transition between frailty states depends highly on one's preceding frailty state [10]. For example, people who are non-frail or pre-frail are more likely to maintain or transition to a robust state than those already in a frail state. Therefore, early detection is critical for preventing or reversing frailty.

For older adults who are not frail yet but developing frailty, monitoring their behaviroal changes could provide insight into how frailty was developed [11]. Conventional measures such as standardized functional tests may not capture such early behavioural changes because those tests are administered Dunder hypothetical or experimental conditions rather than older adults' real lives. Thus, assessing changes in real life may help identify frailty early [11].

Technologies may offer cost-effective opportunities for assessing frailty in older adults' real-life such as home environments. Recent developments in pervasive computing and smart home technology for health monitoring have shown opportunities to monitor signs of frailty onset at home. Researchers have tested various sensors to measure frailty criteria or performance in physical tests for assessing frailty [12]. However, research on technologies for assessing frailty at home is still in its infancy. Most existing systems and approaches focused on a single dimension of the multidimensional frailty and only on homogenous sensors such as wearables [13-15]. A significant number of frailty criteria and early signs associated with frailty were not studied using heterogeneous technologies in home settings. Examples of these criteria or early signs include sedentary behaviour [16], constricted life space [11], declined Activities of Daily Livings (ADLs) (e.g., stair climbing) [16], or self-report exhaustion [6]. Many of these criteria are measurable by off-the-shelf sensors. For example, wearable sensors, such as Fitbit[17] or force sensors 
[18], can assess sedentary behaviour. Accelerometer and GPS[19] can measure life-space mobility. Also, inertial sensors[20-22] or cameras[23] can measure ADLs like chair stand or stair climbing. Additionally, previous studies have used speech recognition technology to capture health data for cancer, diabetes, or heart failures [24]. Similar technology could also collect self-report data for assessing frailty.

Moreover, while Internet connectivity and remote access should become an essential design requirement for remote health monitoring systems $[25,26]$, most existing systems for assessing frailty lack telecommunication capability, making the systems not convenient and scalable for real-life home deployment. Modern technologies such as the Internet of Things (IOT) and Cloud Computing have demonstrated their capabilities to overcome the remote access barrier in many health care applications (e.g., telemedicine, medical imaging, public health, and patient self-management) [27].

In this paper, we introduce a home-based frailty assessment system named Frailty Toolkit (FT). FT adopts the following design principles to fill some of the gaps identified above:

- FT consists of a unique and new collection of heterogeneous off-the-shelf sensors tailored for homebased frailty assessment. The sensors measure early behavioural signs of frailty in older adults' daily lives.

- FT uses state-of-the-art cloud services from major commercial cloud service providers. The cloud technologies enable real-time telecommunication and the use of advanced data analytics.

- FT makes no use of cameras or wearable sensors. Such devices are perceived to have privacy and obtrusiveness concerns, undermining user acceptance. Instead, ambient sensors require minimal effort from end-users to monitor frailty. Users are not required to carry any device.

- The design of FT invovles older adults' voices from the beginning to enhance its usability.

The overall objective of FT research is to adapt off-the-shelf sensors for use in homes and evaluate the system's effectiveness in assessing frailty. The specific objective of this paper is to validate the concurrent validity of measuring frailty criteria using multiple sensors in a simulated home lab environment.

The rest of this article is structured as follows: Section 2 presents the design method and experiment protocol. Section 3 reports the statistical results. Section 4 discusses our study with guidance for future work. Finally, Section 5 presents the conclusions.

\section{Methods}

\section{Material}

\subsection{Frailty Criteria and Sensor Selection}


The sensor selection for FT was based on both frailty criteria defined in existing clinical frailty scales and phenotypes that were found significantly associated with frailty by previous studies. We used Fried's frailty phenotype model [6] as the primary reference clinical model. We also referenced the cycle of frailty theory [28] and phenotypes that are significantly associated with frailty, such as life-space mobility [11] and ADLs [17]. Combining frailty criteria from different models and domains may help build a new frailty assessment paradigm tailored for home-based frailty assessment. Accordingly, we can identify effective off-the-shelf sensors to measure those criteria or phenotypes. We chose ambient sensors instead of wearable or vision-based sensors because ambient sensors are non-invasive and preserve privacy.

\subsubsection{Strength}

Handgrip strength was one of the five phenotypes defined in Fried's frailty model. However, the hand dynamometer's rare availability even in primary care settings, absence of remote communication, and inadequate user-friendliness limit its usage in the home by older adults without assistance [29]. To find a home-friendly sensor to measure strength, we further looked into the cycle of frailty theory shown in Figure 1, which defines a progressing cycle and a broader range of interrelated frailty phenotypes that include the five criteria in Fried's phenotype model. We found that the strength and immobilization belong to the same progressing cycle (see Figure 1). Moreover, another study found that immobilization was associated with sedentary behaviour [30]. As sedentary behaviour is associated with frailty [16], it may be an alternative underlying strength indicator for off-the-shelf sensors to measure at home. We, therefore, designed a mat sensor to monitor sedentary behaviour instead of straight measuring handgrip strength.

In addition to sedentary behaviour, strength can also be measured by physical performance tests such as standing balance, chair stand, and stair climb [31]. Among these tests, stair climbing time was significantly associated with the early onset of frailty [17]. Like sedentary behaviour, stair climbing can also be measured by off-the-shelf sensors more easily than handgrip strength in an unsupervised home environment. In this study, we chose to use ultrasonic distance sensors to detect human presence on a flight of stairs for calculating stair climbing time. We used two distance sensors and placed one at the first and last step of a flight of stairs respectively to capture the start and end of a stair-climbing event.

\subsubsection{Self-report Exhaustion}

The self-report exhaustion data is collected by a customized Raspberry Pi-based smart speaker. The smart speaker was programmed to collect users' speech through a 6-mic audio board (ReSpeaker 6-Mic Circular Array Kit for Raspberry Pi, Seeed Technoogy Inc.). The smart speaker used a customized cloudbased Amazon Lex chatbot to manage the conversation with its users. Amazon Lex is an Amazon Web Service that provides automatic speech recognition and natural language understanding technologies to create a Speech-Language Understanding system or a chatbot. The Lex chatbot was built to ask two questions from the Center for Epidemiological Studies-Depression (CES-D) scale [32] for the self-report exhaustion data collection. UUnlike the commercial smart speakers such as Google Home or Amazon Echo that must be triggered using a keyword from a user, the speaker built in this study would initiate a conversation when other sensors in FT detect a subject. The smart speaker would ask the following two 
questions sequentially "Do you feel that everything you did was an effort?" and "How often in the last week did you feel this way?". While the first question is a "yes" or "no" question, the second question expects users to answer one of the three words, "always," "sometimes," or "rare." Users who respond "sometimes" or "always" will be categorized as frail for this criterion. A sample interaction between the smart speaker and a user is illustrated in Figure 2.

\subsubsection{Physical Activity}

Fried's phenotype model uses calorie consumption per week to measure physical activity. Technologies such as smartwatches or computer vision can measure calorie consumption or estimate consumption by identifying activities or food [33,34]. However, these technologies either require high compliance from users or violate user privacy. Instead of measuring calorie consumption, we proposed to use motion sensors to measure gross movement at room levels through the number of sensor triggers. Information such as presence duration in a room (e.g., bedroom, living room) and frequency of room transitions can be obtained. An earlier study has shown the potential of using room transition data to distinguish frailty statuses [35]. The study used Bluetooth beacons placed in each room and a smartphone carried by users to detect room transitions. This study used motion sensors as they may be a more effortless and unobtrusive alternative to capture similar information. Each functional room has one motion sensor installed.

\subsubsection{Weight}

Weight loss can be easily measured by tracking weight changes using a bathroom scale. To facilitate the use of a low-cost home bathroom scale, we modified a standard digital bathroom scale by adding an Arduino-based microcontroller with a wireless communication module and a LED light. With the modification, the scale can initiate and prompt the weight measuring process by working with other sensors in FT and send weight data to the loT platform. For example, once the motion sensor in the bathroom detects a person's presence, the smart weight scale would receive a command from the motion sensor immediately to blink the LED light prompting the start of the weight measuring process. The smart weight scale was factory calibrated as the weighing module was not modified.

\subsubsection{Life Space Mobility}

Life space is one of the behavioural precursors of frailty, as a large cohort study found that women who left the neighbourhood less frequently were 1.7 times more likely to become frail than those who left the neighbourhood four or more times per week [11]. To estimate life space from home without using wearable technology like GPS, we used a simple door sensor installed at the entrance door of a home to monitor home entry and exit. Thus, parameters associated with life space, such as frequency and duration of being away from home within a specific time frame can be estimated. Table 1 shows the final selection of sensors. 
Table 1

Sensors' hardware components, corresponding frailty criteria.

\begin{tabular}{|ll|}
\hline Sensor & Frailty Criteria \\
\hline Mat sensor & Strength through sedentary behaviour \\
\hline Distance sensor & Strength through stair climbing performance (ADL) \\
\hline Smart speaker & Self-report exhaustion \\
\hline Motion sensor & Physical activity, life space mobility (indoor) \\
\hline Door sensor & Life space mobility (outdoor) \\
\hline Smart weight scale & Weight \\
\hline
\end{tabular}

\subsection{User-centred Design}

Deploying technologies into older adults' homes faces many challenges caused by older adults' physiological impairments, stigma concerns, obtrusiveness, or technical barriers [36]. The technology design process should involve older adults and other stakeholders to understand their living habits and home environments, their preferred ways of interacting with the technologies, and their preferred functionalities and deployment process [37]. We conducted focus group interviews with older adults before the start of the design [37] and adopted the following practical design recommendations learned from the focus groups.

Each sensor in FT operated autonomously after powering on. No further interventions were needed. The software running on the sensors could be updated remotely without user interaction. The only requirement from the users was to recharge the batteries or plug in the power adapter. This minimal effort from the users was positioned to reduce the user-perceived complexity of the system and potential operating errors.

Moreover, the smart speaker in FT could be placed in a convenient location according to the older adults' lifestyles. For example, some older adults preferred to interact with the speaker in the kitchen while preparing food. Others chose to put the speaker near the bed for a quick conversation before sleeping. In addition, to enhance usability, the smart speaker would play a soft prompting ringtone before the frailty conversation, resembling the one used in the airport before any announcements. At the end of the conversation, the speaker would confirm all information was received and appreciate users' responses. Additionally, we gave users feedback on the smart weight scale by using a LED light to show weight measurement progress. When the loT platform received the weight data successfully, the LED light turned green to tell the user that the measurement was complete.

Other design considerations that have not been incorporated into the current system but will be in the following design iteration include:1) adding physical buttons to the smart speaker for those older adults who prefer the familiar, simple button press to the verbal conversation with the smart speaker. The goal 
was to reduce the complexity or probability of confusion to enhance usability; 2) use existing technologies such as smartphones to improve data collection and communication. The use of smartphones, in this case, does not mean older adults have to carry smartphones as the earlier studies did [38,39]. Instead, the under-used smartphone or other existing technologies at home could be reused to enhance data collection in a user-familiar way, such as text messaging or app notification. For instance, an Al-powered text messaging chatbot can ask self-report questions and get user responses by text messages. Information about frailty status, abnormal patterns in sensor data, and help information can also be shared by text messages or an app notification; 3 ) adding warming functionality into the mat sensor to enhance technology enjoyment; 4) adding enjoyable functions to the smart speaker, such as playing music and telling jokes [37].

\section{Protocol}

As this study aims to validate the sensor measurements but not frailty assessment results, a convenience sample of healthy young participants is sufficient to achieve the goal. A convenience sample of nine healthy young adults was recruited by sending group emails to research labs and posting flyers in hospitals of the University Health Network (UHN), Toronto. The recruitment lasted from May to August 2021. Participant's inclusion and exclusion criteria are as follows:

\section{Inclusion criteria}

- Minimum 18 years old

- Able to understand and speak English

- Able to give informed consent

- Able to attend at least one experiment session

\section{Exclusion criteria}

- Have trouble getting in and out of bed without assistance

- Have trouble walking or always use a wheelchair

- Cannot speak due to speech impairment

- Cannot hear due to hearing impairment

Participants' demographic information was collected to determine their eligibility. Each participant attended one experiment session in a simulated home called HomeLab at KITE Research Institute of Toronto Rehabilitation Institute, UHN, Toronto, Canada. The HomeLab is a "home within a lab", which resembles a typically furnished single-storey one-bedroom apartment with functional plumbing and wiring. Figure 3 illustrates the HomeLab layout and sensor setup. 
Each participant performed a series of daily activities during the experiment session to test pre-installed sensors in HomeLab. These activities include room transitions (to test motion sensors), main entrance entry and exit (to test door sensor), stair climbing (to test distance sensors), weight measuring (to test smart weight scale), sitting (to test mat sensor), and exhaustion question answering (to test smart speaker).

Each participant completed three runs of the experiment. Each run of the experiment took at least 15 minutes. In the first run, each participant was given detailed verbal instructions by a researcher (CB) on the activities to be performed. The instructions include going to a particular room (e.g., living room), measuring weight using the smart weight scale, climbing a flight of stairs, sitting on a chair with a mat sensor, and having a conversation with the smart speaker. Participants were then asked to perform similar activities based on their own decision and pace in the second and third runs. A summary of the experimental protocol is shown in Table 2. Participants were required to perform the activities defined in the protocol at least once. It was common that participants repeated these activities multiple times at different times. These activities triggered the sensors to generate sensor data. Depending on the sensor type, the data could contain information related to the room where a participant is present (e.g., living room or dining room), weight, occupancy status for the first or last step of the flight of stairs, and chair occupancy, or door opening status. All data were transmitted to a cloud-based loT platform and immediately timestamped when stored in a cloud database attached to the loT platform. The sensor data were then transferred to a server at the UHN, Toronto, Canada.

All experiment sessions were video and audiotaped. The video and audio recordings were used to extract ground truth data for the activities performed by each participant except for the weight. The weight ground truth data were manually collected using a traditional non-digital weight scale in HomeLab at the end of each session. All activities were performed under the supervision of a researcher (CB). During the experiment, the researcher was not in HomeLab but stood at a catwalk overhanging around the lab with a bird's-eye view of the lab. Break periods were preserved between different test runs. 
Table 2

Experiment Protocol for Testing the Sensors in FT.

\begin{tabular}{|c|c|c|c|}
\hline Run & Run \#1 & Run \#2 & Run \#3 \\
\hline Run Type & $\begin{array}{l}\text { Guided, normal } \\
\text { pace }\end{array}$ & $\begin{array}{l}\text { Self-paced, } \\
\text { normal }\end{array}$ & $\begin{array}{l}\text { Self-paced, slow (mimicking frail older } \\
\text { adults) }\end{array}$ \\
\hline Activity Type & \multicolumn{3}{|l|}{ Activities } \\
\hline Physical activity & \multicolumn{3}{|c|}{$\begin{array}{l}\text { Go to a room (e.g., living room) in HomeLab and do whatever activities in the } \\
\text { room for } 2 \text { minutes. }\end{array}$} \\
\hline $\begin{array}{l}\text { Sedentary } \\
\text { behaviour }\end{array}$ & \multicolumn{3}{|c|}{ Sit on a chair that has a mat sensor. } \\
\hline $\begin{array}{l}\text { Weight } \\
\text { measuring }\end{array}$ & \multicolumn{3}{|c|}{ Measure weight using the smart weight scale. } \\
\hline Stair climbing & \multicolumn{3}{|c|}{ Climb a flight of stairs. } \\
\hline $\begin{array}{l}\text { Self-report } \\
\text { exhaustion }\end{array}$ & \multicolumn{3}{|c|}{ Have a conversation with the smart speaker. } \\
\hline Life space & \multicolumn{3}{|c|}{ Enter or exit HomeLab through the main entrance door. } \\
\hline
\end{tabular}

\section{Data processing}

The sensor measurement for each frailty criterion was compared with the corresponding ground truth measurement (video or audio recordings). The agreements between the two approaches were assessed using the Cohen's Kappa test and Bland Altman plots. The Cohen's Kappa test was used for categorical data from the motion sensor, whereas the Bland Altman plots were used for continuous data such as sedentary time and stair climbing time. Bivariate correlation was used to analyze weight data. All statistical tests were performed by the Statistical Package for Social Sciences (SPSS Inc., Chicago, IL, USA). The interpretation of agreement strength for Cohen's Kappa was following Landis and Koch's guidelines (1977) [40]. For Bland Altman plots, more than $20 \%$ of the value that fell outside the limits of agreement (LoA) would be considered no agreement between the two methods. P values less than 0.05 were considered to be statistically significant.

\section{Results}

The three repeated runs of the experiment from nine participants generated 580 activities in total.

\section{The smart speaker for self-report exhaustion}


The smart speaker recognized and recorded participants' answers to the two questions from the CES-D Depression scale. The recognized answers by the smart speaker had a 100\% agreement with those recorded in the audio recordings $(n=27)$.

\section{The motion sensor for room-level physical activity}

Cohen's Kappa was used to analyze the agreement in room-level physical activity measurements between motion sensors and video recordings. The two methods generated a kappa value of $k=0.938$ which showed an almost perfect agreement. The kappa value was significantly different from zero $(k=0.938$, $p<0.001)$.

\section{The door sensor for life-space mobility}

The door sensor had an $87.5 \%$ successful rate in detecting a door entry/exit event. However, the five undetected door entry/exit events were caused by a technical issue in the door sensor and solved immediately during the experiment. Therefore, the accuracy reaches $100 \%$ when excluding these outliers. See Table 3 of the percentage agreement.

Table 3

Frequency and percentage of successfully detecting door entry/exit event by the door

sensor.

\begin{tabular}{|lll|}
\hline & Frequency & Percentage \\
\hline Detected & 35 & 87.5 \\
\hline Undetected & 5 & 12.5 \\
\hline Total & 40 & 100 \\
\hline
\end{tabular}

\section{The mat sensors for sedentary behaviour}

The Bland Altman plot was used to assess the agreement of the data from the mat sensor $(n=42)$ and distance sensor $(n=38)$ with their corresponding ground truth data extracted from the video recordings.

Figure 4 shows the plot of sedentary time duration between the two methods. The plot shows a mean difference, also called bias, of -0.286 , presenting as a solid, purple line. LoA is shown as the area between the two solid, green lines with $95 \%$ LoA. Only $4.8 \%$ (2 out of 42) values fall outside the LoA, suggesting a good agreement between the two methods [41, 42]. As Figure 4 shows, the values are uniformly spread 
below and above the bias/mean difference within the LoA, suggesting no proportional bias. The result is also confirmed by linear regression $(t=1.097, p=0.279)$.

\section{The distance sensors for stair climbing time}

The distance sensor had a 50\% successful rate in detecting a stair-climbing event, including climbing up or down a flight of stairs, as shown in Table 4. The detected stair climbing time and ground truth time were compared using the Bland Altman plot. Figure 5 reports a mean difference of 0.526 between the two measurements, shown as a solid, purple line. LoA is shown as the area between the two solid, green lines with 95\% LoA. The majority of the values are inside the LoA, suggesting there is a good agreement between the two methods. As Figure 5 shows, the values are uniformly spread below and above the bias within the LoA, suggesting no proportional bias. The result is also confirmed by linear regression $(t=0.653$, $\mathrm{p}=0.518)$.

Table 4

Frequency and percentage of successfully detecting stair-climbing event by the distance sensors.

\begin{tabular}{|lll|}
\hline & Frequency & Percentage \\
\hline Detected & 38 & 50 \\
Undetected & 38 & 50 \\
\hline Total & 76 & 100 \\
\hline
\end{tabular}

\section{The smart weight scale for weight}

There is a significant difference in the data generated from the two measurements. This could probably be caused by the incorrect operation or inaccurate readings of the traditional weight scale. However, bivariate correlation shows a strong, positive correlation between the two measurements, $r=0.942, n=24$, $p<0.001$ (see Figure 6). This suggests that the smart weight scale could be used to track an individual's weight change.

\section{Discussion}

The FT has been designed to capture behavioural and physical health changes in daily life that are related to frailty. The use of a novel combination of ambient sensors, smart speaker, weight scale, and cutting-edge loT and cloud technologies made the system highly autonomous and minimally dependent on users' memories and compliances. This research is an essential foundational work in determining the value of a sensor-based in-home frailty monitoring system with the ultimate goals of supporting older 
adults to remain at home as long as possible and enhancing their quality of life. This phase of the research tested the concurrent validity of the measurements of the proposed sensor system. A sensor system that has proven concurrent validity will lay the groundwork for the next phase of testing using the system in older adults' homes.

Although most of the sensors showed excellent agreements with their respective gold standard measurements, there were missed detections from the motion sensors in the experiments. Most of the missed detections came from the first two participants. This was because of the hardware glitches, such as loose connections, that were not found in the dry run, but were revealed at the beginning of the experiment with the first two participants. After the fix was done, no glitches were found for the rest participants. If the first two participants were excluded from the analysis, the agreements should be higher.

Moreover, the distance sensor had only a $50 \%$ successful rate in detecting a stair-climbing event. We found that the missed detection was because some participants used one side (e.g., left) of the stair to climb, whereas the sensors were installed on the opposite side (e.g., right). This caused the distance between the participants and the sensors to be beyond the distance threshold $(60 \mathrm{~cm})$, which did not trigger the sensors.

Regarding the smart speaker, its role in FT can be extended well beyond the data collection for self-report exhaustion. The smart speaker opens up new possibilities for collecting data from a multidimensional perspective around physical activity, food intake, life space, and more frailty indicators. The technical capabilities of the smart speaker also allow more functions that older adults like to add in the future. For example, more enjoyment features, such as playing music, can be integrated into the chatbot to enhance its usability. In addition, the smart speaker can be more helpful by giving users prompts or reminders about their health changes identified by FT and recommending possible interventions.

One strength of the study is that the design of FT involved older adults' voices from the beginning, which has not been found in other similar technologies for assessing frailty. Flexible sensor placement, simplified human-computer interaction, LED light prompting were some of the recommendations learned from the focus group study and have been incorporated in the FT design. Another strength from the technical perspective is that we have successfully implemented and tested the "trigger-action" mechanism between the IoT sensors to improve system usability and enhance the precision of data collection for particular sensors such as the smart weight scale and smart speaker. With the mechanism, the triggering of a motion sensor or a mat sensor can further activate a smart weight scale prompting users for weight measuring or a smart speaker for initiating a conversation for collecting self-report exhaustion data, respectively. This inter-sensor communication capability is the first time being applied in similar systems. Lastly, participants were given opportunities to perform activities in HomeLab based on their paces freely. The freedom allowed the system to be tested in different scenarios that are closest to real life. 
FT also has its limitations. It can only work for single-person dwellings as no sensors in the current FT can distinguish different persons. Indeed, the Radio Frequency Identification technology can be used to provide a unique ID for identifying different people. Computer vision technology can also provide personal identity through face recognition. However, both technologies can bring compliance or privacy concerns, making them not ideal for an application with older adults in home settings. Moreover, as FT is a proof-of-concept system, the current energy management for each sensor is not ideal for extending the battery life. The improvement in battery life can be made by removing unused electronic components on the current micro-controllers or using more energy-efficient micro-controllers. Also, mat and distance sensors can only use time but not event signals to control their deep sleep mode. Unnecessary power waste using time-based sleep mode can shorten battery life. A comparator circuit with relays and logic gates could be one way to improve to allow pin interrupt-based sleep control.

\section{Future work includes:}

- Test the system in HomeLab with non-frail and frail participants. The test would allow more data collection for training a machine learning model for classifying non-frail and frail older adults.

- Move the system from the simulated home to an actual home of an older adult who lives alone. While older adults continue to live their own lives, the system will continuously run for a week to collect data. The data will be compared with the older adult's frailty status measured by a reference clinical frailty scale.

- Develop sensors to measure new frailty criteria or phenotypes in different domains and identify other persons living in the same household.

\section{Conclusion}

This study showed excellent concurrent validity for the ambient sensors and the smart speaker in FT. The smart weight scale strongly correlated with its gold standard measurement, but more validation is needed to confirm its concurrent validity. Overall, FT is reliable for monitoring physical and behavioural signs of frailty in home settings. The choice of sensors and frailty criteria and the involvement of older adults' voices made FT unique to other exiting technologies. The next step of the research is to test the toolkit with frail and non-frail older adults to validate the system's clinical effectiveness.

\section{Abbreviations}

ADLs - Activities of Daily Livings

CES-D - Center for Epidemiological Studies-Depression

FT - Frailty Toolkit

loT - Internet of Things 
LoA - Limits of Agreement

UHN - University Health Network

\section{Declarations}

\section{Ethics approval and consent to participate}

The Research Ethics Board at University Health Network, Toronto, Ontario, Canada, approved this study protocol (protocol code: 20-5806 and date of approval: 26 January 2021). All methods were performed in accordance with the relevant guidelines and regulations stated in the Declaration of Helsinki. Written informed consent to participate have been obtained from each participant prior to the study.

\section{Consent for publication}

Not applicable.

\section{Availability of data and materials}

The datasets generated and/or analysed during the current study are not publicly available due to confidentiality protocol. Data are however available from the corresponding author on reasonable request and subject to approval from the Research Ethics Board at University Health Network, Toronto, Ontario, Canada.

\section{Competing interests}

The authors declare that they have no competing interests.

\section{Funding}

The project is funded by AGE-WELL NCE (Aging Gracefully across Environments using Technology to Support Wellness, Engagement and Long Life NCE Inc.) - Networks of Centres of Excellence of Canada.

\section{Authors' contributions}

The first author (C.B.) designed and developed the sensor system used in the study. C.B. also conducted all the experiments with participants in the lab and wrote the manuscript. The second author (B.Y.) was the lab manager and managed participant recruitment, data, and study documents. She also conducted statistical data analysis and revised the manuscript. The third author (A.M.) made a significant 
contribution in reviewing the study protocol for Research Ethics Board, revising the manuscript, and providing academic and financial sponsorship to the study. All authors read and approved the final manuscript.

\section{Acknowledgements}

The authors would like to extend our sincere thanks to all study participants, for their time and contributions. We are also grateful to staff at the KITE Machine Shop at KITE Research Institute, Toronto Rehabilitation Institute, for their help in machining sensor enclosures.

\section{Author details}

KITE Research Institute, Toronto Rehabilitation Institute, University Health Network, Toronto, Canada

Chao Bian, Bing Ye, \& Alex Mihailidis

Institute of Biomedical Engineering, University of Toronto, Toronto, Canada

Chao Bian \& Alex Mihailidis

Department of Occupational Science and Occupational Therapy, University of Toronto, Toronto, Canada

Bing Ye \& Alex Mihailidis

\section{References}

1. United Nations, Department of Economic and Social Affairs PD. World Population Prospects 2019: Highlights (ST/ESA/SER.A/423). 2019.

2. Polanczyk CA, Marcantonio E, Goldman L, Rohde LEP, Orav J, Mangione CM, et al. Impact of Age on Perioperative Complications and Length of Stay in Patients Undergoing Noncardiac Surgery. Annals of Internal Medicine. 2001;134:637. doi:10.7326/0003-4819-134-8-200104170-00008.

3. Davenport DL, Bowe EA, Henderson WG, Khuri SF, Mentzer RM. National Surgical Quality Improvement Program (NSQIP) Risk Factors Can Be Used to Validate American Society of Anesthesiologists Physical Status Classification (ASA PS) Levels. Annals of Surgery. 2006;243:63644. doi:10.1097/01.sla.0000216508.95556.cc.

4. Makary MA, Segev DL, Pronovost PJ, Syin D, Bandeen-Roche K, Patel P, et al. Frailty as a Predictor of Surgical Outcomes in Older Patients. Journal of the American College of Surgeons. 2010;210:901-8. doi:10.1016/j.jamcollsurg.2010.01.028.

5. Rockwood K, Andrew M, Mitnitski A. A Comparison of Two Approaches to Measuring Frailty in Elderly People. The Journals of Gerontology Series A: Biological Sciences and Medical Sciences. 2007;62:738-43. doi:10.1093/gerona/62.7.738. 
6. Fried LP, Tangen CM, Walston J, Newman AB, Hirsch C, Gottdiener J, et al. Frailty in Older Adults: Evidence for a Phenotype. The Journals of Gerontology Series A: Biological Sciences and Medical Sciences. 2001;56:M146-57. doi:10.1093/gerona/56.3.M146.

7. Maresova P, Javanmardi E, Barakovic S, Barakovic Husic J, Tomsone S, Krejcar O, et al. Consequences of chronic diseases and other limitations associated with old age - a scoping review. BMC Public Health. 2019;19:1431. doi:10.1186/s12889-019-7762-5.

8. Mondor L, Maxwell CJ, Hogan DB, Bronskill SE, Campitelli MA, Seitz DP, et al. The Incremental Health Care Costs of Frailty Among Home Care Recipients With and Without Dementia in Ontario, Canada. Medical Care. 2019;57:512-20. doi:10.1097/MLR.0000000000001139.

9. Hajek A, Bock J-O, Saum K-U, Matschinger H, Brenner H, Holleczek B, et al. Frailty and healthcare costs-longitudinal results of a prospective cohort study. Age and Ageing. 2018;47:233-41. doi:10.1093/ageing/afx157.

10. Gill TM, Gahbauer EA, Allore HG, Han L. Transitions Between Frailty States Among Community-Living Older Persons. Archives of Internal Medicine. 2006;166:418. doi:10.1001/archinte.166.4.418.

11. Xue QL. The Frailty Syndrome: Definition and Natural History. Clinics in Geriatric Medicine. 2011;27:1-15.

12. Bian $\mathrm{C}, \mathrm{Ye} \mathrm{B}, \mathrm{Chu} \mathrm{CH}$, McGilton KS, Mihailidis A. Technology for home-based frailty assessment and prediction: A systematic review. Gerontechnology. 2020;19:1-13. doi:10.4017/gt.2020.19.003.06.

13. Gianaria E, Grangetto M, Roppolo M, Mulasso A, Rabaglietti E. Kinect-based gait analysis for automatic frailty syndrome assessment. Proceedings - International Conference on Image Processing, ICIP. 2016;2016-Augus:1314-8.

14. Jaber R, Chkeir A, Hewson DJ, Duchene J. A New Device to Assess Gait Velocity at Home. IFMBE Proceedings. 2014;41:670-3.

15. Ferre X, Villalba-Mora E, Caballero-Mora M-A, Sanchez A, Aguilera W, Garcia-Grossocordon N, et al. Gait Speed Measurement for Elderly Patients with Risk of Frailty. Mobile Information Systems. 2017;2017:1-11. doi:10.1155/2017/1310345.

16. Del Pozo-Cruz B, Mañas A, Martín-García M, Marín-Puyalto J, García-García FJ, Rodriguez-Mañas L, et al. Frailty is associated with objectively assessed sedentary behaviour patterns in older adults: Evidence from the Toledo Study for Healthy Aging (TSHA). PLoS ONE. 2017;12:1-9.

17. Tuttle LJ, Bittel DC, Bittel AJ, Sinacore DR. Early-Onset Physical Frailty in Adults With Diabesity and Peripheral Neuropathy. Canadian Journal of Diabetes. 2018;42:478-83. doi:10.1016/j.jcjd.2017.12.001.

18. Merry KJ, Macdonald E, MacPherson M, Aziz O, Park E, Ryan M, et al. Classifying sitting, standing, and walking using plantar force data. Medical \& Biological Engineering \& Computing. 2021;59:25770. doi:10.1007/s11517-020-02297-4.

19. Zijlstra W, Giannouli E. Mobility in community-dwelling older adults; what are its determinants? BMC Geriatrics. 2021;21:228. doi:10.1186/s12877-021-02174-1. 
20. Ganea R, Paraschiv-lonescu A, Büla C, Rochat S, Aminian K. Multi-parametric evaluation of sit-tostand and stand-to-sit transitions in elderly people. Medical Engineering and Physics. 2011;33:108693. doi:10.1016/j.medengphy.2011.04.015.

21. Millor N, Lecumberri P, Gómez M, Martínez-Ramírez A, Izquierdo M. An evaluation of the 30-s chair stand test in older adults: frailty detection based on kinematic parameters from a single inertial unit. Journal of NeuroEngineering and Rehabilitation. 2013;10.

22. Hellmers S, Kromke T, Dasenbrock L, Heinks A, Bauer JM, Hein A, et al. Stair Climb Power Measurements via Inertial Measurement Units - Towards an Unsupervised Assessment of Strength in Domestic Environments. 2018;5 Biostec:39-47.

23. Allin S, Mihailidis A. Low-cost, Automated Assessment of Sit-To-Stand Movement in "Natural" Environments. In: IFMBE Proceedings. Springer Berlin Heidelberg; 2009. doi:10.1007/978-3-54089208-3_20.

24. Bérubé $C$, Schachner T, Keller R, Fleisch E, v Wangenheim F, Barata F, et al. Voice-Based Conversational Agents for the Prevention and Management of Chronic and Mental Health Conditions: Systematic Literature Review. Journal of Medical Internet Research. 2021;23:e25933. doi:10.2196/25933.

25. Piau A, Charlon Y, Campo E, Vellas B, Nourhashemi F. A Smart Insole to Promote Healthy Aging for Frail Elderly Individuals: Specifications, Design, and Preliminary Results. JMIR Rehabilitation and Assistive Technologies. 2015;2:e5. doi:10.2196/rehab.4084.

26. Dasios A, Gavalas D, Pantziou G, Konstantopoulos C. Hands-On Experiences in Deploying CostEffective Ambient-Assisted Living Systems. Sensors. 2015;15:14487-512. doi:10.3390/s150614487.

27. Griebel L, Prokosch H-U, Köpcke F, Toddenroth D, Christoph J, Leb I, et al. A scoping review of cloud computing in healthcare. BMC Medical Informatics and Decision Making. 2015;15:17. doi:10.1186/s12911-015-0145-7.

28. Xue QL, Bandeen-Roche K, Varadhan R, Zhou J, Fried LP. Initial manifestations of frailty criteria and the development of frailty phenotype in the women's health and aging study II. Journals of Gerontology - Series A Biological Sciences and Medical Sciences. 2008;63:984-90.

29. Jaber R, Chkeir A, Hewson D, Duchene J. ARPEGE: Assessment of frailty at home. 2013 IEEE 15th International Conference on e-Health Networking, Applications and Services, Healthcom 2013. 2013; Healthcom:434-8.

30. Charansonney OL, Després J-P. Disease prevention-should we target obesity or sedentary lifestyle? Nature Reviews Cardiology. 2010;7:468-72. doi:10.1038/nrcardio.2010.68.

31. Nightingale EJ, Pourkazemi F, Hiller CE. Systematic review of timed stair tests. Journal of Rehabilitation Research and Development. 2014;51:335-50. doi:10.1682/JRRD.2013.06.0148.

32. Orme JG, Reis J, Herz EJ. Factorial and discriminant validity of the Center for Epidemiological Studies Depression (CES-D) scale. Journal of clinical psychology. 1986;42:28-33. doi:10.1002/10974679(198601)42:1<28::aid-jclp2270420104>3.0.co;2-t. 
33. Fuller D, Colwell E, Low J, Orychock K, Tobin MA, Simango B, et al. Reliability and Validity of Commercially Available Wearable Devices for Measuring Steps, Energy Expenditure, and Heart Rate: Systematic Review. JMIR mHealth and uHealth. 2020;8:e18694. doi:10.2196/18694.

34. Myers A, Johnston N, Rathod V, Korattikara A, Gorban A, Silberman N, et al. Im2Calories: Towards an Automated Mobile Vision Food Diary. In: 2015 IEEE International Conference on Computer Vision (ICCV). IEEE; 2015. p. 1233-41. doi:10.1109/ICCV.2015.146.

35. Tegou T, Kalamaras I, Tsipouras M, Giannakeas N, Votis K. A Low-Cost Indoor Activity Monitoring System for Detecting Frailty in Older Adults. 2019.

36. Doyle J, Bailey C, Ni Scanaill C, van den Berg F. Lessons learned in deploying independent living technologies to older adults' homes. Universal Access in the Information Society. 2013. doi:10.1007/s10209-013-0308-1.

37. Bian C, Ye B, Hoonakker A, Mihailidis A. Attitudes and perspectives of older adults on technologies for assessing frailty in home settings: a focus group study. BMC Geriatrics. 2021;21:298. doi:10.1186/s12877-021-02252-4.

38. Galán-Mercant A, Cuesta-Vargas Al. Clinical frailty syndrome assessment using inertial sensors embedded in smartphones. Physiological measurement. 2015;36:1929-42.

39. Fontecha J, Navarro FJ, Hervás R, Bravo J. Elderly frailty detection by using accelerometer-enabled smartphones and clinical information records. Personal and Ubiquitous Computing. 2013;17:107383.

40. Landis JR, Koch GG. The Measurement of Observer Agreement for Categorical Data. Biometrics. 1977;33:159. doi:10.2307/2529310.

41. Krakowiak P, Walker CK, Tancredi DJ, Hertz-Picciotto I. Maternal Recall Versus Medical Records of Metabolic Conditions from the Prenatal Period: A Validation Study. Maternal and Child Health Journal. 2015;19:1925-35. doi:10.1007/s10995-015-1723-0.

42. Pursey K, Burrows TL, Stanwell P, Collins CE. How Accurate is Web-Based Self-Reported Height, Weight, and Body Mass Index in Young Adults? Journal of Medical Internet Research. 2014;16:e4. doi:10.2196/jmir.2909.

\section{Figures}




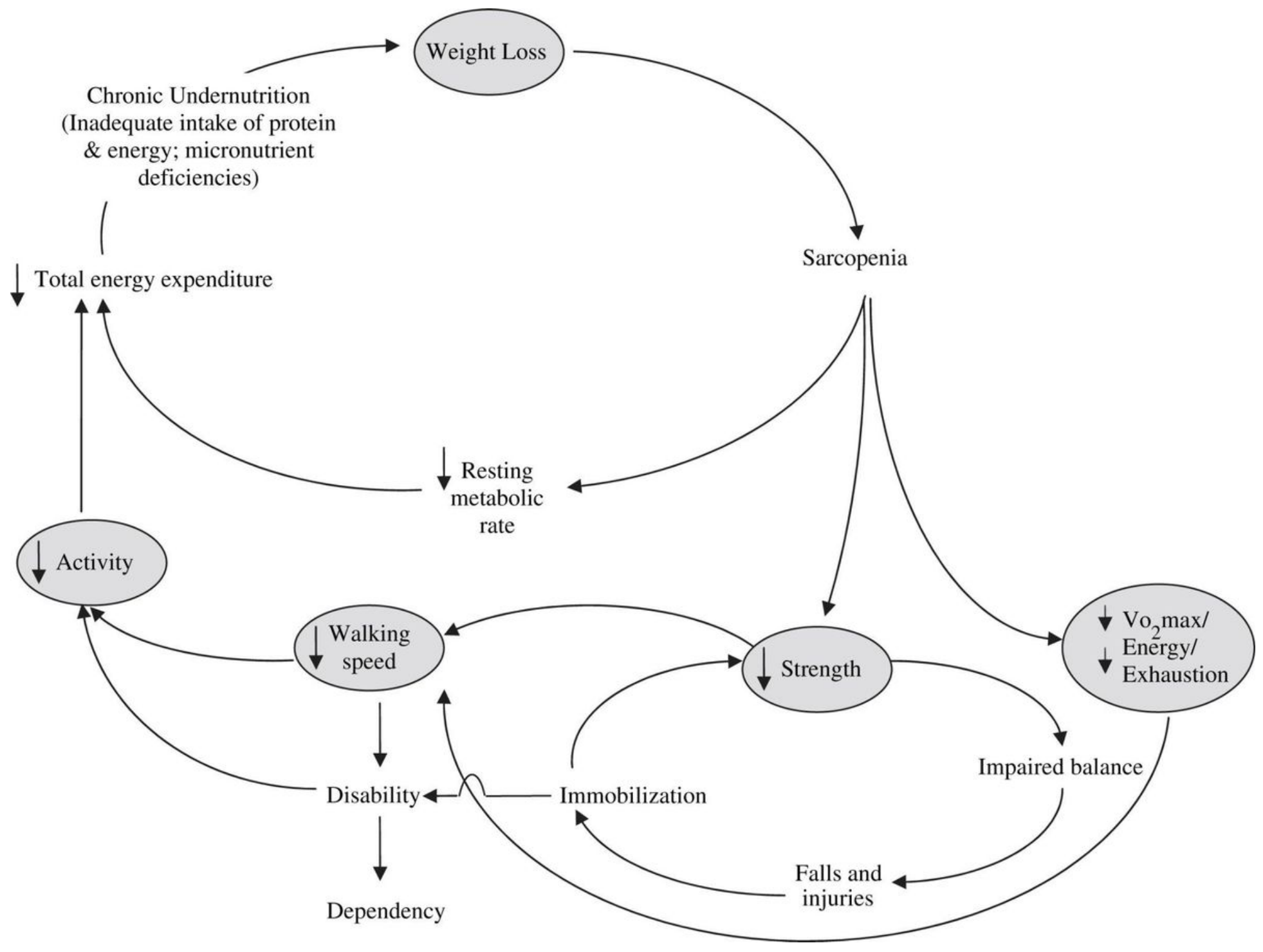

Figure 1

The cycle of frailty [29]. 
Play a soft prompting ringtone

(p))

Hello, this is frailty toolkit. Would you like to answer a few questions about your health?

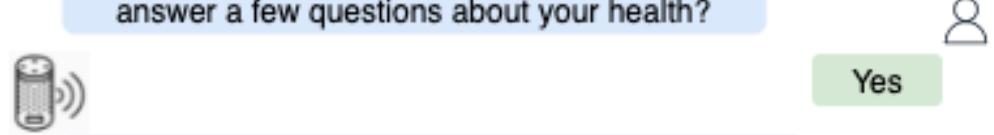

Do you feel that everything you did was an effort?

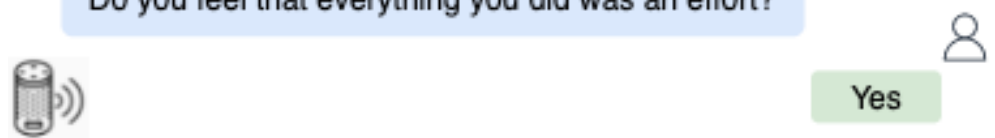

How often in the last week did you feel this way? Please say always, sometimes, or rare.

@))

Thank you for your answers. Have a great day!

\section{Figure 2}

Sample smart speaker conversation. 


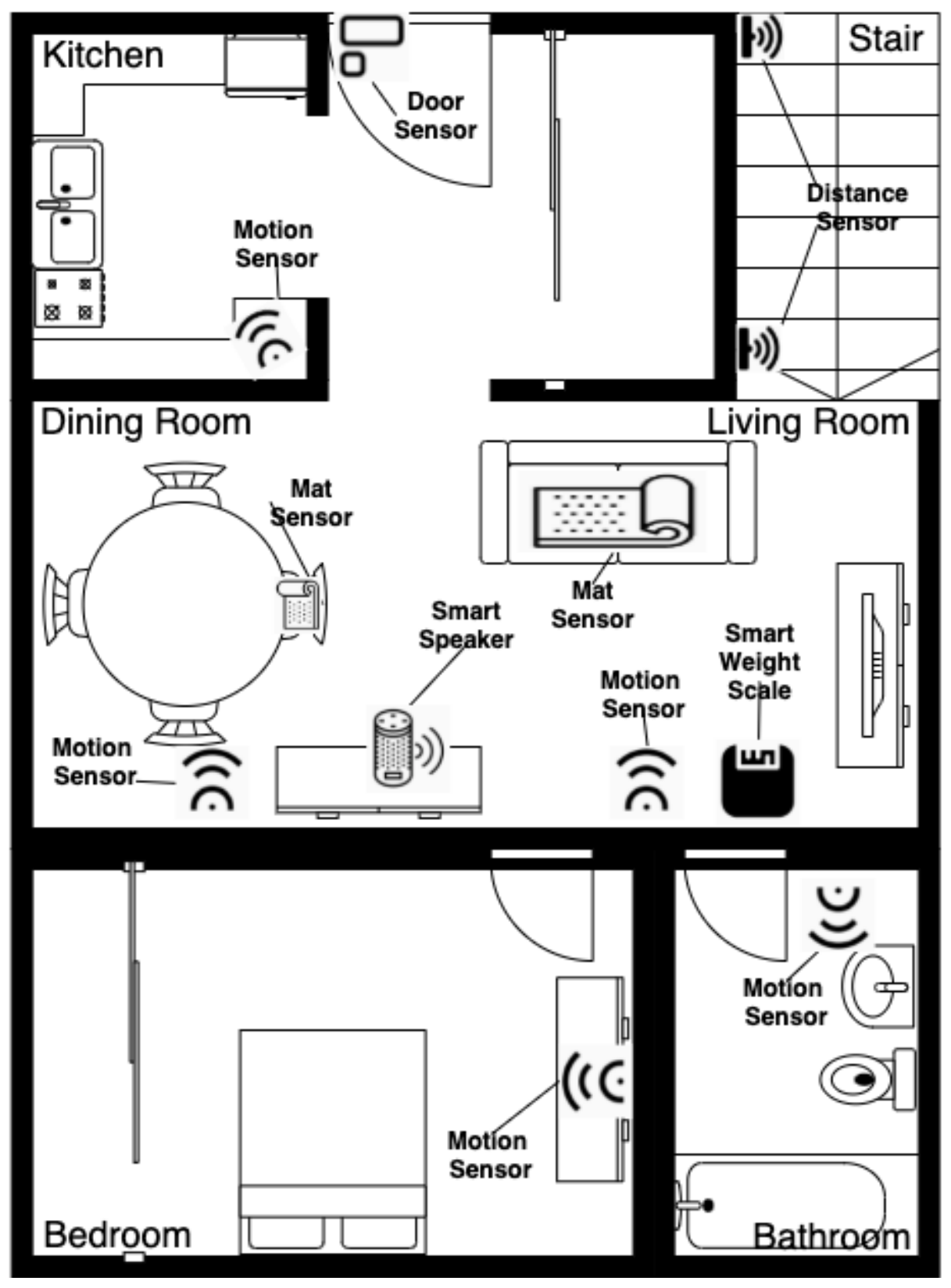

Figure 3

Sensor setup layout in HomeLab. 


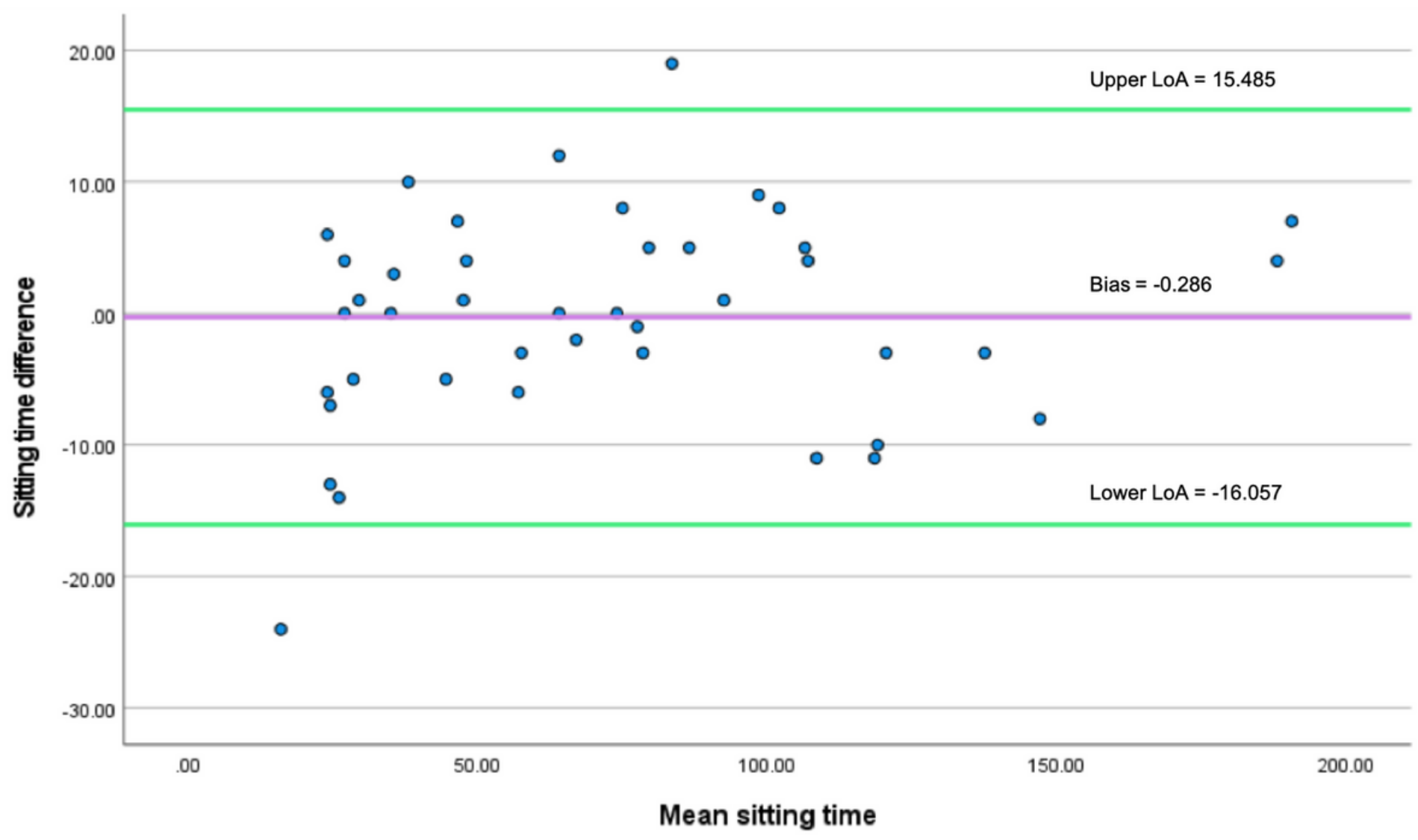

\section{Figure 4}

The Bland Altman plot of agreement between mat sensor and video recording.

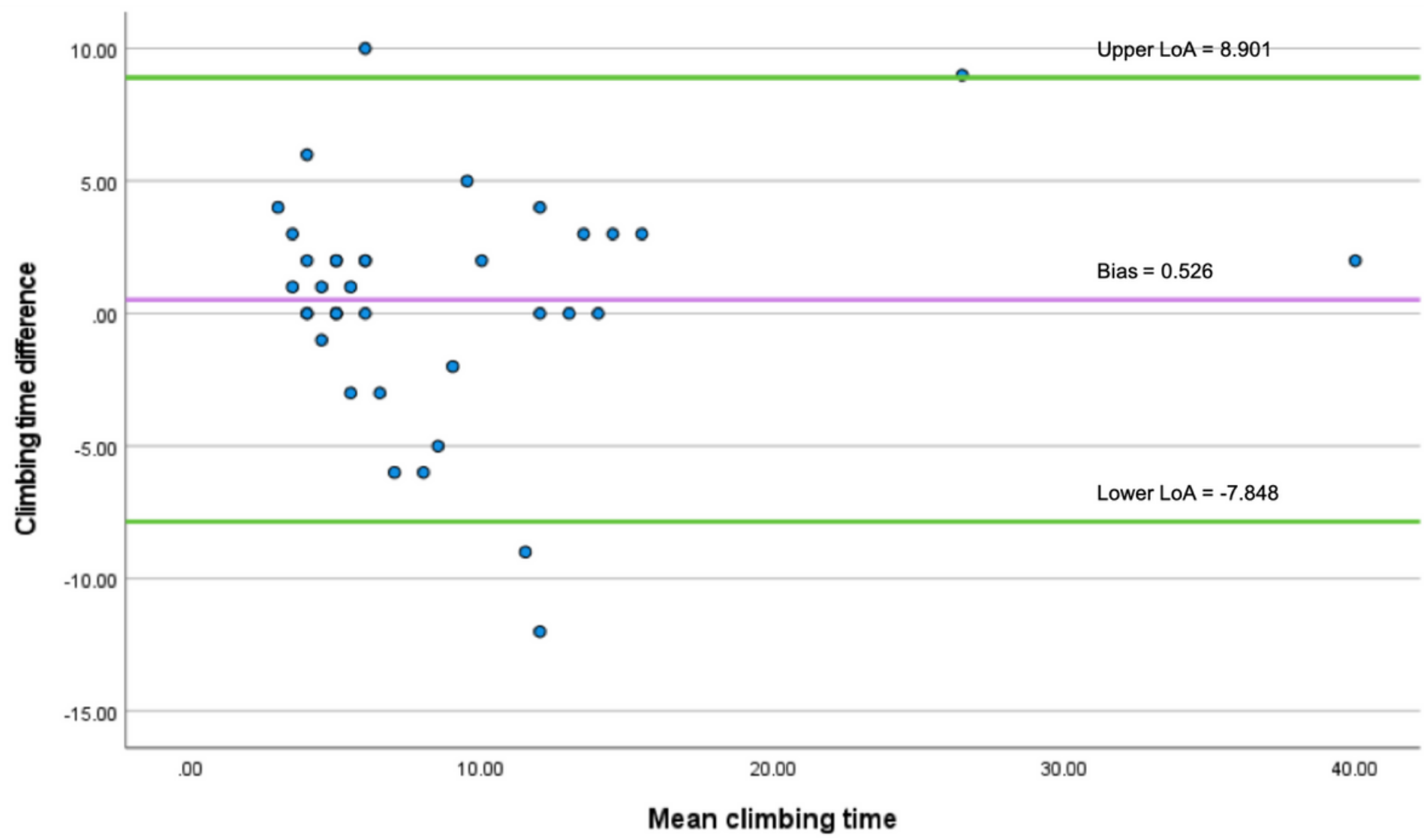

Page 23/24 


\section{Figure 5}

The Bland Altman plot of agreement between distance sensor and video recording.

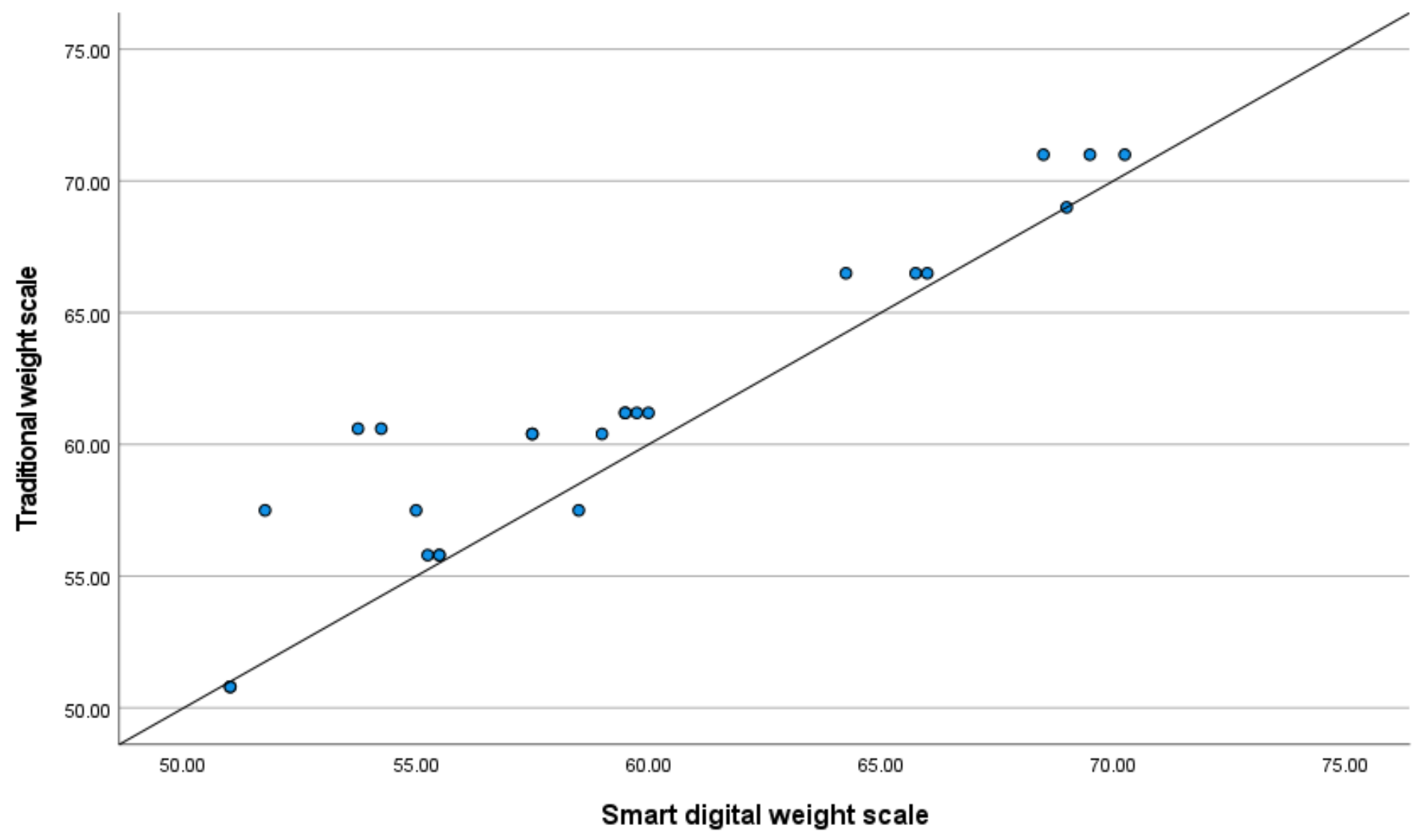

Figure 6

Scatterplot of the weight measured by traditional weight scale against smart digital weight scale. 